\title{
Aeronautical Data Networks
}

\author{
Mustafa Cenk Erturk, Wilfrido Moreno, Jamal Haque and Huseyin Arslan \\ University of South Florida \\ USA
}

\section{Introduction}

The wireless connectivity is becoming an integral part of our society. The advances in signal processing, rapid prototyping and an insatiable consumer demand for wireless connectivity is opening a new paradigm of data service, "Aeronautical Data Networks (ADN)". Programs lead by National Aeronautics and Space Administration (NASA), Federal Aviation Administration (FAA) [NASA/CR-2008], EUROCONTROL and Networking the Sky for Civil Aeronautical Communications (NEWSKY) [Newsky] are all including the aeronautical platform as part of their network. The objective is to provide a low delay and cost effective data network for an aeronautical platform, as well as use it as a relay for ground and airborne nodes [Sakhaee], [Medina]. Most of current systems use a satellite for connecting to an aeronautical platform. Satellite resources are limited, expensive and offer limited data throughput as compared to a terrestrial networks. Moreover, frequency spectrum is a valuable estate and needs to be used efficiently. Hence, advance spectrum efficient techniques needs to be evaluated for this environment.

The book chapter will explore the challenges of aeronautical environment to provide connectivity at all times. A detail analysis with mathematical equations will be presented to show the aeronautical channel impairments. The impact of Doppler on the channel that limits the use of a highly efficient modulation scheme, such as orthogonal frequency division multiplexing (OFDM), will be presented. Doppler has a major impact on OFDM based systems. In addition, Doppler spread in ADN depicts rather different characteristics compared to terrestrial networks, i.e., multiple Doppler shifts in the channel and profound delays. Results of parametric spectrum estimation methods for extracting the Doppler shifts will be presented.

OFDM in combination with dense encoding, offers a robust communication and spectrum compression, however its usage is limited to terrestrial domain due to Doppler. OFDM sensitivity to frequency shifts results in intercarrier interference (ICI) and degrades spectral efficiency. High mobility platform, such as train and aircraft offer a challenging environment for OFDM. OFDM ICI and frequency shift caused by the high mobility of the platform is investigated and potential methods are proposed.

ADN's can provide a critical service for various situations, such as: public safety communications, denial of service (DoS), disaster situations, in-flight Internet, as well as mobile communication on the ground such as providing services for highways, trains etc. The network connectivity of ADN will be explored. Current and future prospects of ADN will be discussed in terms of cross interoperability with a terrestrial backbone. The result of 
a notional network capacity analysis is presented. Connectivity and robustness of an Aeronautical based Network, both as a relay for terrestrial networks and to provide in-flight internet will be presented.

Finally the chapter will explore the system and architecture requirements for a cognitive driven reconfigurable hardware for an aeronautical platform, such as commercial aircraft or high altitude platforms. The scope of such a system would provide an intelligent configurable radio system, provide connectivity for a changing geographical, political and regulatory environments that an aircraft experiences. Such a system will take advantage of opportunistic services available for today and future. With advances in components and processing hardware, mobile platforms such as those mentioned above are ideal candidates to have configurable hardware that can morph itself, given the location and available wireless service. The global movement of the aeronautical system can take advantage of emerging wireless services and standards. This section of the chapter will propose a system for an intelligent self-configurable software and hardware solution for an aeronautical system, Cognitive Aeronautical Software Defined Radio (CASDR).

\section{Motivation and challenges}

The ever-changing geographical environment of an aircraft and an increasing availability of different wireless services make's one wonder, what if such services can be accessed in real time.

This provided the motivation to develop a concept system and its hardware that would accommodate to the rapid changes, not just due to the aircraft location, but also to support the growth of services and industry evolution. Fig. 1 depicts the notional framework of opportunistic wireless data service that may be available for an aircraft in flight. At higher altitude the services may be more traditional and fixed, however on ground, the growing WiMAX and local area network services may be available to be accessed from the aircraft. The high-speed mobility of an aircraft adds additional challenges to the design of system physical layer, such as path loss and multi-Doppler spread.

\section{Literature review}

The desire for a universal and a reconfigurable terminal first appeared in the military area. The need for mobility and accessibility was the driving requirement. One of the early concept was a reconfigurable system appeared as an equipment called "SPEAKeasy". The Software Communications Architecture (SCA) developed by the Joint Tactical Radio System (JTRS) program of the U.S. Department of Defense (DoD) further fueled the growth of SDR. JTRS aims to provide a family of digital, programmable, multiband, multimode, modular radios to alleviate communications interoperability problems. Finally the work of J. Mitola [Mitola_1], there is now a growing interest in reconfigurable terminals.

The increase in air traffic is resulting in the surge of commercial airborne communication system [Eurocontrol]. Aircell and AeroSat have developed the ground based hardware and now offer in flight Internet service. Aircell uses a concept of air-to-ground link [Bluemenstein] and provides the in-flight Internet service called 'gogo' on aircrafts. GOGO service works of cellular phone base stations in the continental US, which act as access points for an en route flight. A recent flight from Tampa, Florida to Detroit, Ohio USA, a user using GOGO service experienced an average upload speed of $0.27 \mathrm{Mbits} / \mathrm{s}$ and an 


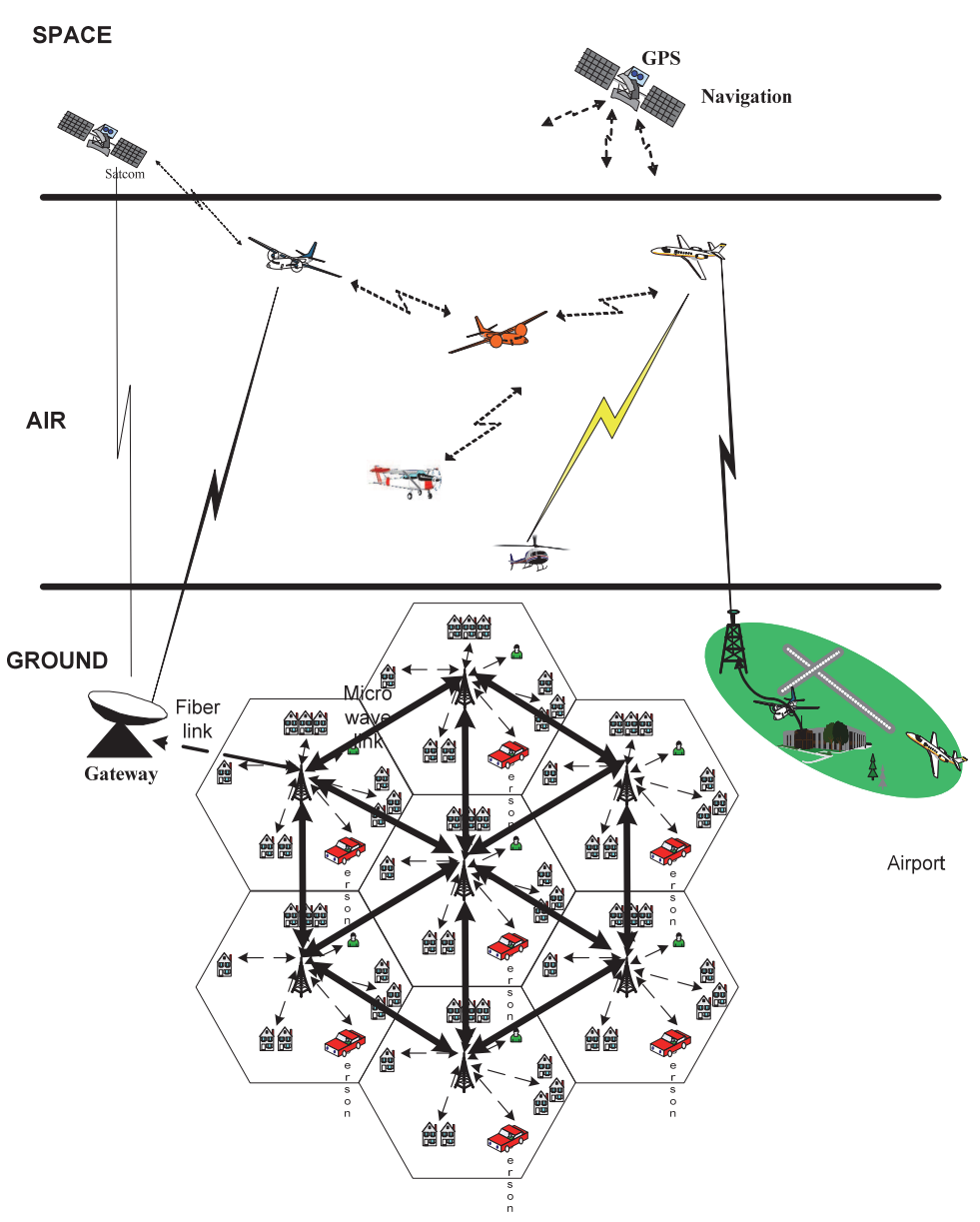

Fig. 1. Aeronautical System

average download speed of $0.33 \mathrm{Mbits} / \mathrm{s}$ with latency of $233 \mathrm{~ms}$. However, the ground based service is limited to flight coverage over land only. For the oceanic flight satellite based connectivity is required. AeroSat developed satellite communication (SATCOM) Ku band for commercial airliners [AeroSat]. This offers broad connectivity, however the cost and data throughput of satellite based service is not conducive to user demand.

The growth in SDR has been enabled by advances in semiconductor, which has led to the development of programmable multi-core General Purpose Processor (GPP), Digital Signal Processor (DSP), Field Programmable Gate Array (FPGA) and Analog to Digital Converter (ADC). GPP, DSPs and FPGAs provide the programmability and processing capability to realize such a system. Hence, the processing chain starting from digital intermediate frequency (IF) down to demodulation can be implemented in digital signal processing [Srikanteswara], [Mohebbi]. Another key enabler is the high speed ADC that bridges the analog and digital world [Zanikopoulos], [Salkintzis]. Advance algorithms that require intense processing can now be implemented in the combination of these moderate size, weight and power processing 
engines. FPGA's, with their ability to parallelize, can implement intense processing algorithms that may be difficult to implement in a DSP or GPP.

Therefore the maturity of; SDR algorithm's, high bandwidth processing engines, development of tunable antenna and availability of high speed ADC makes the implementation of CASDR a possibility. The global mobility of an Aeronautical platform is the ideal implementation of a CASDR concept. A CASDR will learn and configure itself in order to provide multi standard/service modem's as it traverses continents, countries and cities.

\section{Aeronautical system}

\subsection{ASDR system scope}

The scope of this system would be to provide an intelligent configurable radio system, provide connectivity for a changing geographical, political and regulatory environments that an aircraft experiences. Such a system will take advantage of opportunistic services available today and planned in future.

The communication design is beginning to converge on standard building blocks, or systems, which form the basic building block of a communication system, i.e., Read Solomon, Turbo Encoder, Modulations, Viterbi etc. Whether a communication link is being developed for short range, long range, line of sight (LOS) or non line of sight (NLOS) the basic building blocks of communication system are the same. If available in software they can be stitched together to build a radio transceiver. Aeronautical Networks (ANs) could be an important application of such systems, since different regions or countries assign different frequency bands based on their needs and spectrum allocation policies.

\subsection{Aeronautical network geometry}

Geometric relations are observed between an aircraft station (AS) or an aircraft's altitude (h1) with a Ground Station (GS). The LOS communication distance (without considering Fresnel and other parameters) from AS to GS can be calculated using the Pythagoras theorem as follows:

$$
d_{1}=\left(h_{1} \times\left[2 R+h_{1}\right]\right)^{0.5} \cong\left(2 R h_{1}\right)^{0.5}
$$

where, $R$ is the radius of the Earth which varies from $6336 \mathrm{~km}$ to $6399 \mathrm{~km}$, but assumed 6370 $\mathrm{km}$ (for the purpose of calculations). For distances between the two nodes above the sea level, the above formula needs additional steps for calculating the communication distance. The formula is calibrated by a statistically measured parameter by International Telecommunication Union (ITU), i.e., ' $k$ '.

$$
d_{1} \cong\left(2 R k h_{1}\right)^{0.5}
$$

Figure 2 shows the maximum communication distances that can be achieved between AS and AS/GS. The jump in the first $2 \mathrm{~km}$ altitudes for GS communications can be considered a very low orbit AS which can reach a communication zone of $D=120 \mathrm{~km}$. Many commercial planes flying at the altitude of $9 \mathrm{~km}$ can potentially create communication zones about $\mathrm{D}=250 \mathrm{~km}$ with a very conservative approach $(\mathrm{k}=0.5)$. On the other hand, considering the communication distance between two ASs, it can be inferred that it could reach up to $\mathrm{D}=480 \mathrm{~km}$ with $\mathrm{k}=1 / 2$. 


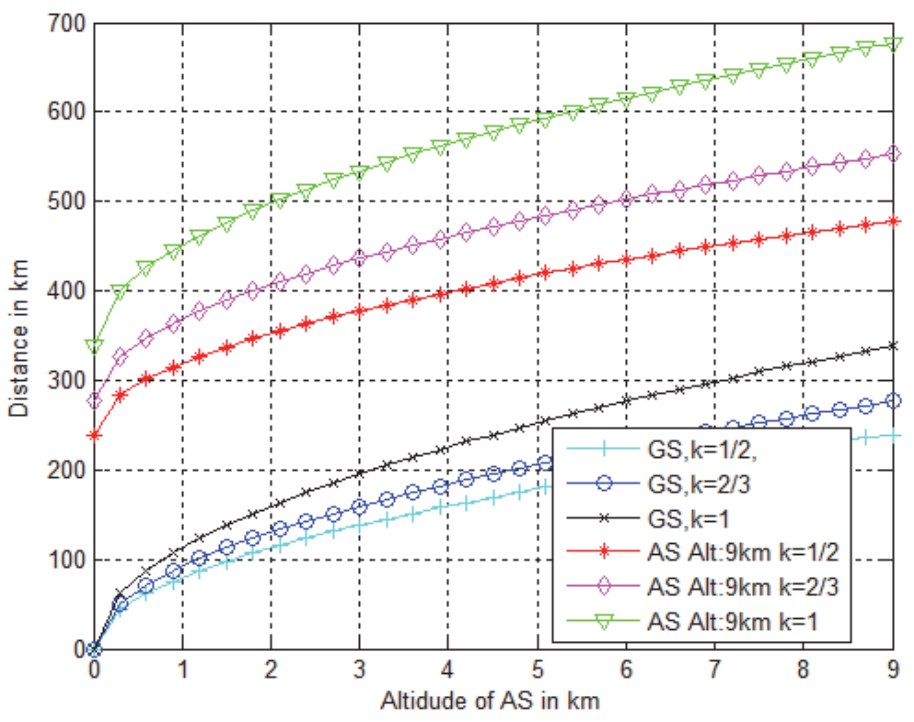

Fig. 2. Communication zone of an AS

Figure 2 shows that ASs could be used as a backhaul or relay for wireless infrastructures, since they have the capability of communicating long distances as compared to wireless ground backhauls. Aeronautical Network (AN) will have a substantial lower round trip delay, which will allow for a low delay telephone and voice over IP services.

\subsection{Aeronautical network scenarios and data access}

Aeronautical Networks can provide critical services for various situations, such as; public safety communications, Denial of Service (DoS), disaster situations, in-flight Internet, as well as mobile communication on the ground, providing services for highways, trains, etc. The network structure that is being proposed in this paper is as follows: Given a particular region to be covered, initially Service Gateway Ground Stations (SGGS) should be built according to the communication distance, see Fig. 3. Assuming that a GS can communicate to an AS within the distance of $200 \mathrm{~km}$, roughly 8 SGGS will be able to provide service for an area of $1600 \mathrm{~km}$ by $800 \mathrm{~km}$.

Data access in an AN can be defined as follows: When a GS or AS has data to send, the flow of the data should be from/to SGGS so the connection with other networks such as public switched telephone network (PSTN), cellular networks and Internet Protocol (IP) could be established.

To provide in-flight services, a centralized configured network should be considered; SGGSs act like Base Stations (BS), covering a particular region where Subscriber Stations (SS) are simply ASs. Scheduling is done by the SGGS and in this structure, AS's are not communicating with each other, except with SSGS's. However, if an AS is not able to register to a SGGS, which could be a case of oceanic flights, then data of that particular AS should be routed to an AS which was already registered to a SGGS with ad-hoc networking strategies. 


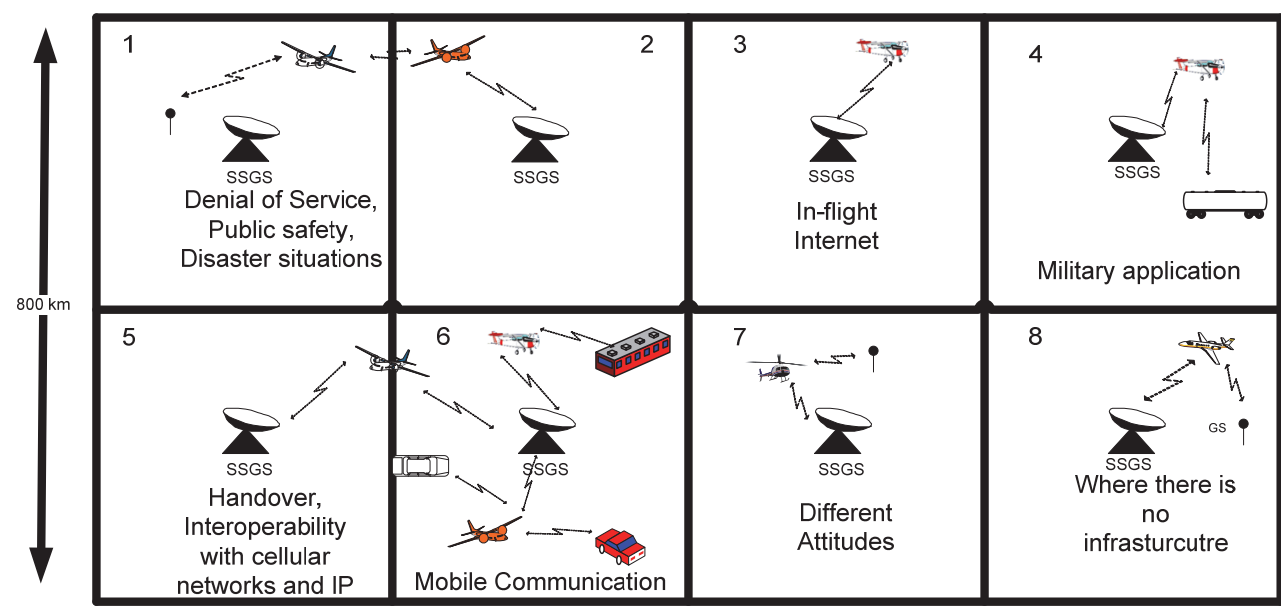

Fig. 3. Aeronautical Network Scenarios

For an AN network the use of AS as a base station used for cellular network is also discussed. In this case, SS's are the GS's, which can be fixed or mobile. When a GS has data to send, it sends its data to an AS. This can be considered as a relay, reflecting the data to its associated SGGS to finalize the establishment. This structure is feasible to provide public safety services in disaster scenarios, provide backhaul option for terrestrial networks and military communication applications. Moreover, in this structure, since both AS's and GS's are not fixed, the handover of a GS between multiple AS is also another challenging issue. It is important to note that the handover process in this structure is somehow different, since GS are doing handovers not only because of their own mobility, but also due to mobility of AS's.

One of the main issues in AN's is the topology estimation. Since there are many mobile stations, in terms of GS and AS, the scheduling and routing of data would differentiate from time to time. In these cases the topology estimation of the network should be done properly, so that the data can be routed and scheduled in mesh and centralized networks strategies respectively.

\subsection{Physical layer}

In a wireless system design, understanding the limits and bounds of a channel impairments theoretically and empirically are critical to the design of the system. An aeronautical environment poses a daunting task to cover a huge area for any system designer. Global channel characteristics need to be understood to establish model parameters. However, this would lean toward statistical average and will result in inefficient system parameters. Current system based on 'gogo' service, uses a ground based link and provides a limited data rates. A data connectivity sample was taken for a Delta flight traversed between Tampa, Florida to Detroit, Ohio USA, using 'Speed Test' (www.speedtest.net). Different 
global servers were pinged periodically duration the flight to measure download, upload and latency. Fig. 4 and Fig. 5 are the global data rates and latency experienced during the flight.

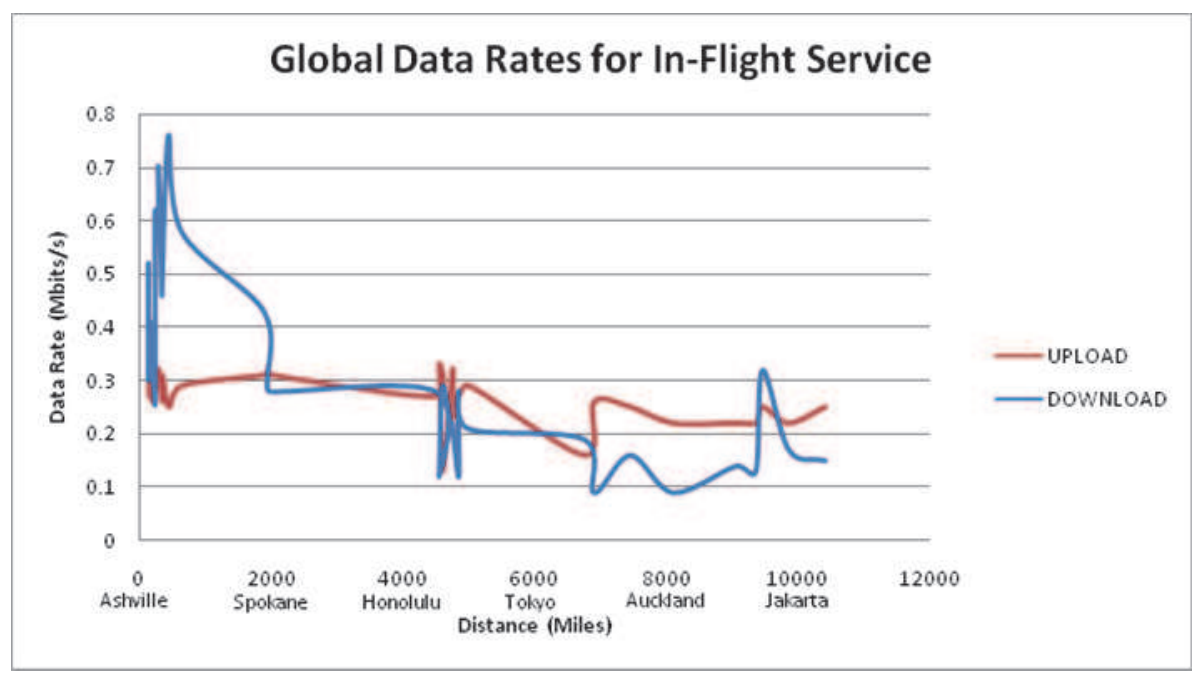

Fig. 4. Global In-Flight Data Rates

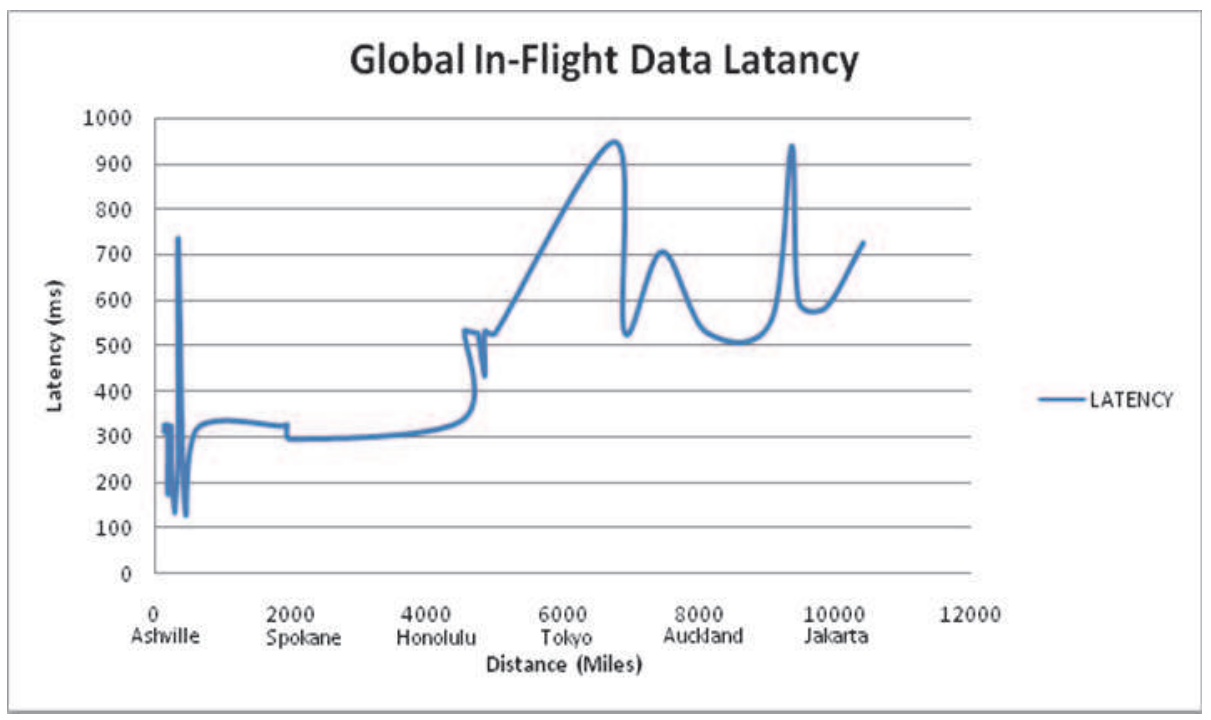

Fig. 5. Global In-Flight Latency

Most of the current system, assumes a line of sight (LOS). This is also the case for the aeronautical platforms connectivity modeling. However, an intelligent CASDR will allow 
for the ability to configure the system and learn the channel condition over the flight route and establish history, hence establish accurate channel parameters for a given location, altitude and speed. Since the aircraft traverses pre-planned route, over time this channel parameters will provide accurate characteristics [Bello]. This will allow higher order spectral efficient modulations and multi-carrier system to be used and provide higher data throughput. Details of this cognitive channel sensing behavior are discussed in section 4 .

A time varying wireless impulse response is represented by equation (3), where the signal is impaired by amplitude, phase, Doppler and time delay.

$$
h(n)=\frac{1}{\sqrt{N}} \sum a_{k} \exp \left(\frac{j 2 \pi \theta}{N}\right) \exp \left(\frac{j 2 \pi f_{d} n}{N}\right) \delta\left(t-\tau_{k}\right)
$$

where $a_{k}, \theta, f_{d}$ and $\tau_{k}$ are amplitude, phase, Doppler shift and delay for each path respectively.

For a LOS, the effect of number of paths is significantly less, $\tau \approx 0, f_{d}$, Doppler shift based on platform would be fixed and a limited variation of phase will lead mostly to amplitude degradation due to path loss. For the diffused path, according to Bello [Bello], it represents a wide-sense stationary uncorrelated (WSSUS) channel, which emulates a small area characterization for multipath channel. The effect of Doppler to the line of sight is mostly frequency shift; however the diffused and specular reflections will have a spread due to Doppler. This Doppler spread for an aeronautical communications depicts a bandwidth less than 360 [Hoeher], [Haas], [Elnoubi]. Most of the current research assumes a two-ray model as the channel model for flat surface areas. In an extremely mountainous terrain environment, the channel model results in an intermittent loss of LOS along with increasing angle spread that could match the Jakes Doppler spread. In the two limiting cases; the angular spread at the receiver depicts either a two ray model or Jakes spectrum. Therefore, a modified Doppler spread model needs to be developed, that will go from a narrow beam width to $360^{\circ}$, as the mobile moves from flat to rough environments. Hence, the use of $D_{f}$ factor from 0 to 1 for the growing Doppler spread, due to beam width, represents going from flat to rough terrain environment:

$$
\begin{gathered}
D_{f}=\frac{\theta_{H}-\theta_{L}}{2 \pi}, \text { where }\left(\theta_{H}-\theta_{L}\right) \leq 2 \pi \\
D=\left\{\begin{array}{cc}
2 D_{f} & , \theta_{H}>\theta>\theta_{L} \\
\infty & , \text { else }
\end{array}\right\}, \text { where } \theta=\cos ^{-1}\left(\frac{f_{d} \lambda}{v}\right),\left|f_{d}\right| \leq f_{d \max }
\end{gathered}
$$

Doppler density going from non-isotropic to isotropic:

$$
p_{f d}\left(f_{d}\right)=\left\{\begin{array}{cc}
\frac{1}{\pi D f_{d \max } \sqrt{1-\left(\frac{f_{d}}{f_{d \max }}\right)^{2}}} & \text { if }\left|f_{d}\right| \leq f_{d \max } \\
0 & \text { else }
\end{array}\right\}
$$




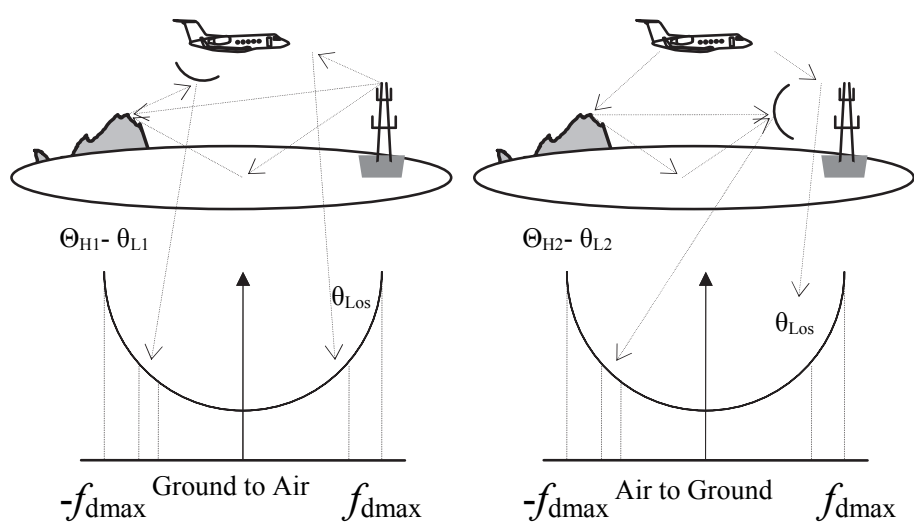

Fig. 6. Doppler Power Spectrums for ADNs

Fig. 6 shows the Air to Ground (A/G) and Ground to Air (G/A) aeronautical communications in an en-route scenario and their corresponding Doppler spectrum. The arrival/take-off, taxi and parking scenarios depicts different multipath and received angle spreads [Haas].The spectrum in an en-route scenario depicts a Doppler shift with a narrow beam Doppler spread, where it can be assumed as another Doppler shift. Among carrier and modulation systems, orthogonal frequency division multiplexing (OFDM) is the most sensitive to Doppler. OFDM based systems has been adopted/proposed for several current/future communication systems all over the world, i.e., asymmetric digital subscriber line (ADSL) services, IEEE 802.11a/g/n, IEEE 802.16, IEEE 802.20, digital audio broadcast (DAB), digital terrestrial television broadcast (DVD) in Europe, ISDB in Japan and fourth generation (4G) cellular systems. Therefore, it is reasonable to assume that any SDR application will also need to support OFDM in the ADN network. In an OFDM based systems, a serial symbol stream is converted into parallel streams and each symbol is modulated with different orthogonal sub-carriers. With the usage of cyclic prefix $(\mathrm{CP})$, since OFDM based systems have already relatively longer symbol durations compared to single carrier systems, they are known for their robustness against frequency selectivity of the channel, i.e., delay spread. However, longer symbol durations lead to weakness of the OFDM systems to time variation of the channel, i.e., Doppler shift/spread which is a challenging issue in ADN.

The two Doppler shifts affecting the system can be described as follows:

$$
y(n)=x(n) * h(n)+w(n)
$$

where, $w(n)$ is noise and $h(n)$ is the channel impulse response defined as:

$$
h(n)=\sum_{i=1}^{2} a_{i} \exp \left(\frac{j 2 \pi f_{\Delta i}\left(n-\tau_{i}\right)}{N}\right) \delta\left(n-\tau_{i}\right)
$$

where $a_{i}$ is the attenuation value, $N$ is the number of FFT bins, $\tau_{i}$ and $f_{\Delta i}$ are the delay and the normalized Doppler frequency shift (NDF) for the first and second ray respectively where $f_{\Delta i}=\frac{f_{D i}}{\Delta_{f}}$. 
For the ADN, Figure 4 presents the two-path channel model. In OFDM, as long as the carrier's orthogonality is maintained, then there is no bleeding of energy. Intercarrier interference (ICI) is related to sub-carrier bandwidth and their proportional interference due to Doppler offset. As an example, an estimation of ICI interference for a system with 64 point FFT OFDM symbol, which has a $312 \mathrm{kHz}$ subcarrier bandwidth, is plotted in Fig. 7.

The Fig. 7 shows the ICI error vector contribution due to frequency shift caused by Doppler in a two ray model. At 0.1 fraction of sub carrier frequency the ICI error contribution approaches $-10 \mathrm{~dB}$. To support higher spectral efficiency generally ICI should remain within or less than 0.02 fraction of sub-carrier frequency. This will allow ICI interfering energy to remain well below $-25 \mathrm{~dB}$ allowing higher spectral efficiency.

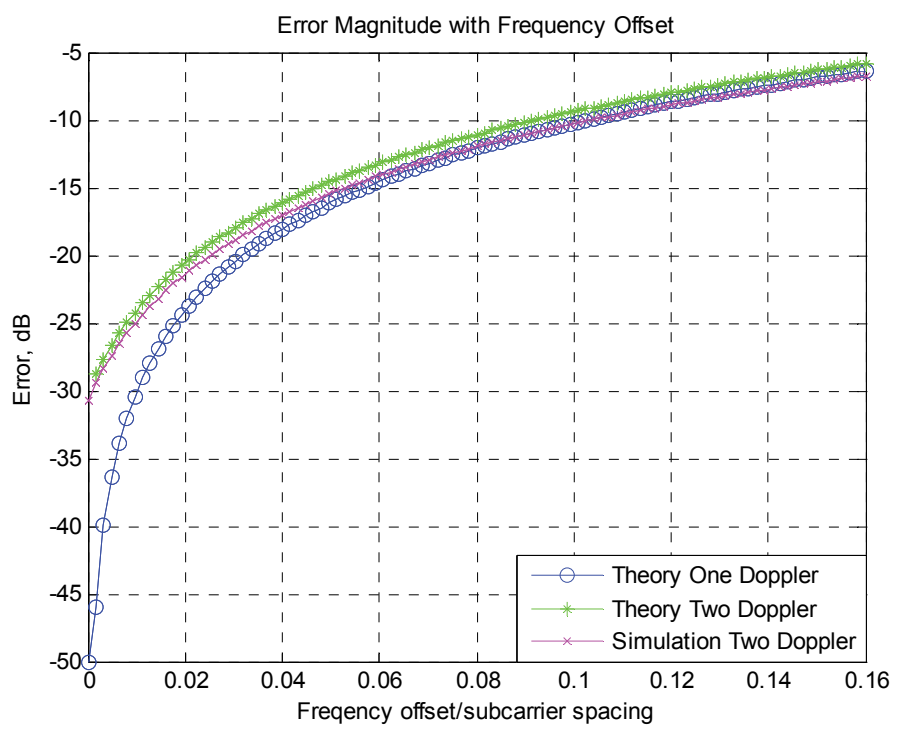

Fig. 7. Doppler ICI vs. Sub-carrier BW

\subsection{Cognitive route based physical layer estimates}

The aircraft routes driven by FAA for various segments are ideal to establish a history of wireless channel conditions for the route. Once a route is traversed, its history of channel impairments are stored with associated coordinates and aircraft attitude information. This data is downloaded to a central database to be shared with another aircraft. For new routes, the cognitive channel estimator will try to understand the channel condition. Over time, the channel history collected from different aircraft will create a channel map for each route. The ASDR will then be able to download this data and prior to a flight adjust the physical layer parameters for the route. For a mobile platform that has a predetermined route, such as AN, the channel estimation is broken down to static and dynamic components. The static components effecting the channel would be large objects i.e., mountains, buildings, etc. The averaging over multiple routes will provide of stable static channel estimates. The dynamic components will be due to time varying objects. 


\section{Aeronautical software define radio}

The advances in components and signal processing techniques are the leading enabler for a configurable hardware and intelligent software. Software defined radio emerges from the desire of single radio hardware that molds its feature to different radio schemes [Apostolis]. The artificial intelligence needed for the smarts of such configurable hardware is emerging into what is known as cognitive radio [Mitola_2].

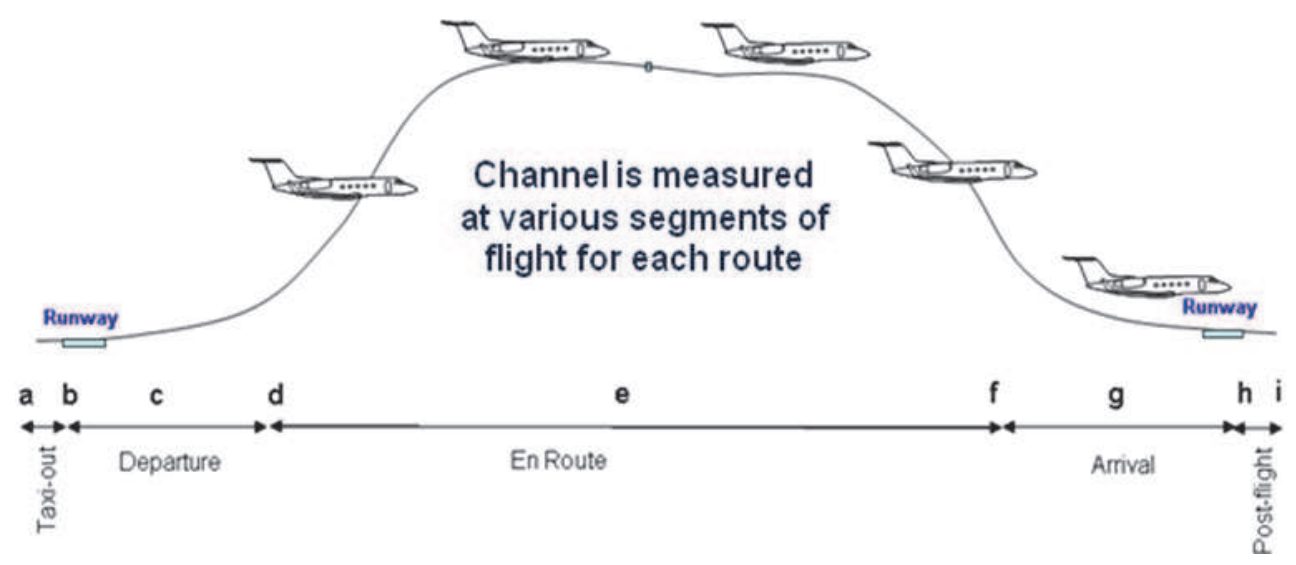

Fig. 8. Route based Channel Sensing

Cognitive algorithms combined with configurable hardware can take full advantage of varying location of an aircraft, whether that is in the air, en route and lends themselves to take advantage of opportunistic spectrum and network connectivity.

A system with the ability to morph to accommodate the aeronautical changing environments, channels conditions across domestics and international boundaries is required. Aeronautical software defined radio (ASDR) platform will also allow the flexibility to comply with countries regulations governing the spectrum usage and interference.

\subsection{Spectrum coverage}

The spectrum bandwidth use and frequency band allocation for different systems is one of the challenges to overcome for truly building an ASDR capable of accommodating itself for different regions. For a given region or country, the standard may be the same but the frequency band used may be different. For example, the 802.16 specification applies across a wide range of radio frequency spectrum and WiMAX could function on any various frequencies i.e., $2.5 \mathrm{GHz}$ is predominantly being used in the USA, elsewhere in the world 2.3 $\mathrm{GHz}$ used in Asia and some countries are using 3.5 GHz.

The Analog TV bands (700 MHz) may become available for WiMAX usage, but currently it is being used for digital TV, however different countries might choose to use the spectrum that best suits their needs. Table 1 below lists opportunistic frequency data network available [Zhang], [Peter]. 


\begin{tabular}{|c|c|c|c|c|}
\hline $\begin{array}{c}\text { Band } \\
(\mathrm{GHz})\end{array}$ & BW $(\mathrm{MHz})$ & Standard & Region & Service \\
\hline 2.4 & 20 & $802.11 \mathrm{~b} / \mathrm{g}$ & US & Wi-Fi \\
\hline 5 & 20 & $802.11 \mathrm{a}$ & US & Wi-Fi \\
\hline 2.5 & 20 & 802.16 & US & Fixed WiMAX \\
\hline $3.5,2.5$ & 20 & $802.16 \mathrm{a}$ & Can & Fixed WiMAX \\
\hline 2.3 & 20 & $802.16 \mathrm{e}$ & Aus & Fixed WiMAX \\
\hline $1.616-$ & 10.5 & Custom & Global & $\begin{array}{c}\text { Iridium Down } \\
\text { Link }\end{array}$ \\
\hline 1.6265 & 20 & Custom & Global & Iridium Up Link \\
\hline $19.4-19.6$ & $1.23,5$ & W/CDMA & EU & 3G Cellular \\
\hline 2,4 & n/a & n/a & & APSK, OFDM \\
\hline $1.9,0.85$ & $1.23,5$ & W/CDMA & US & 3G Cellular \\
\hline $1.8,0.9$ & 1.23 TV \\
\hline $0.5-0.8$ & & & & US \\
\hline
\end{tabular}

Table 1. Wireless Standards

Another feature that will be necessary in a SDR application is a tunable RF front end capable of locking on the various bands.

Frequency bands and bandwidths for future wireless communication studies in terms of aeronautical communications are discussed at the World Radio communication Conference (WRC) 2007. This international body maintains and agrees to abide by the use of spectrum by international treaty. Aeronautical Mobile (Route) Service (AM(R)S) communication is defined as a safety system requiring high reliability and rapid response. Safety and security applications together with, Air Traffic Control (ATC) and Air Traffic Management (ATM) communications are considered to be AM(R)S. To accommodate the future growth of aeronautical communication, new band allocations are being made in AM(R)S rather than VHF band in L and C. L band (960-1164 MHz) and C band (5091-5150 $\mathrm{MHz}$ ) allocations are discussed in the meeting. $\mathrm{L}$ band is suggested as a suitable band for future aeronautical communication studies. $C$ band is considered to be used in Airport surface network systems, since it is thought to be useful for short range, high data throughput.

\subsection{Critical system parameters}

Cognitive radio system will require optimization of system performance. Algorithms capable of real time optimizing the system performance as well as pre/post flight will create pre-flight configuration Table 2; 


\begin{tabular}{|c|}
\hline Aeronautical Optimization Parameters \\
\hline Customer Usage \\
\hline DQ: Quantity of data transferred at various flight segments. \\
$D T:$ Duration of data transfer per segmented route. \\
TC: Traffic classes: multi-media, navigation, system health \& safety. \\
BER: Required Bit error rate per Traffic Classes. \\
Network \& Data Access Layer \\
\hline Protocol Selection, Routing configuration, Forward error selection given the customer \\
driven BER, Available to provide relay service, Packet error rate \\
Physical Layer \\
\hline$T_{M}:$ Multipath delay spread: Characterizes channel smearing due to arrival of multi- \\
signal reflection arrivals. \\
$f_{D S:}$ Doppler spread or Doppler bandwidth. \\
f: Doppler Shift. \\
A: Attenuation: power loss, function of frequency and distance. \\
L: Impulse Response Length: length, in signal elements, of CIR. \\
Band: Carrier frequency Band. \\
BW: Available bandwidth. \\
SWP: Standard waveform performance.
\end{tabular}

Table 2. Parameters

\subsection{Aeronautical cognitive radio}

The term cognitive comes from psychology meaning "brains" the ability to learn and understand. The aeronautical environment is ideal application for an intelligent radio,

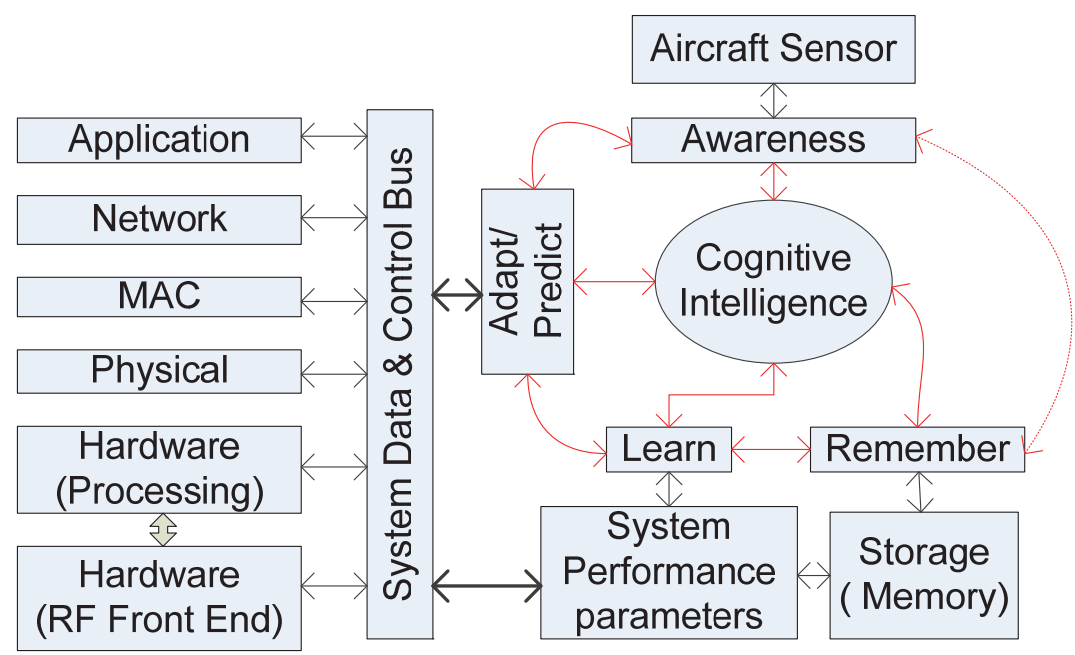

Fig. 9. Aeronautical SDR and CE 
which is capable of learning the environment for various locations and altitudes, see Figure 7 and 8. Over time, each aircraft flying over certain route will store the data on board storage devices. This data shall contain the route the airline/aircraft traversed, the opportunist wireless links available, frequency band, bandwidth, data rate, wireless standard, signal quality for the route, etc. Upon arrival at the destination, data is then downloaded to a centralized flight communication data bank. This data is then available for flights heading on the same route.

\subsection{Aeronautical cognitive intellegence}

The brain of the aeronautical cognitive engine would be to work of its constant awareness of aircraft geographical location and RF channel. It will sense weather conditions that may affect the radio transmission and available services available.

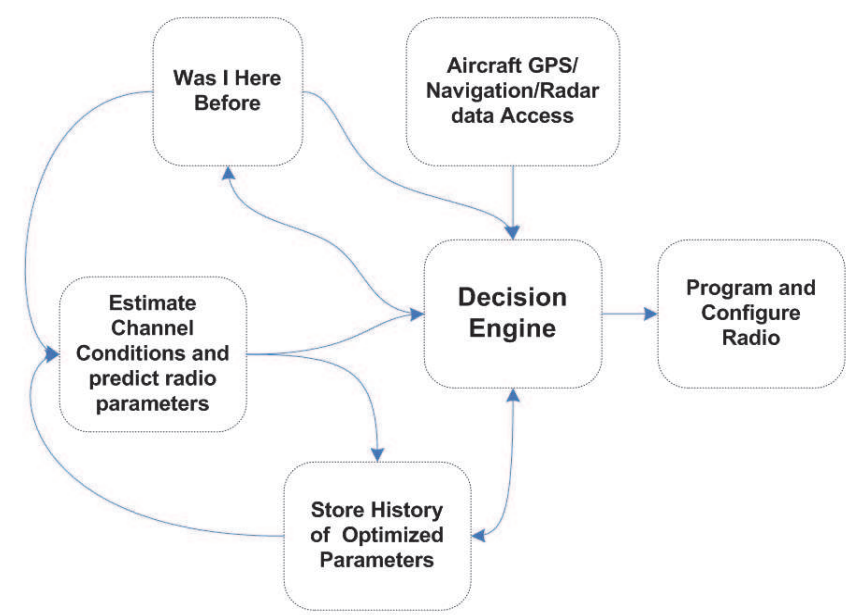

Fig. 10. Cognitive Engine

- Awareness:

The aircraft navigation and radar systems will provide the sensing stimulation to the cognitive engine. The inertial measurement unit (IMU) used for flight navigation will provide aircraft speed, altitude, and attitude. Advance forward looking radar will provide the weather conditions that may affect the radio transmission performance. Global position system (GPS) will provide location of the aircraft with respect to global geography. Furthermore, the awareness engine will have the ability to estimate the data requirements based on past data use and flight profile, before accessing the spectrum for services.

- Learn:

The cognitive awareness provides an opportunity for CASDR to learn the spectrum usage, data demand and system throughput based flight route during day or night. Such statistics will allow a constant learning and developing statistics profile that is stored for each route. This allows cognitive radio of other airlines that have not travelled that particular route to have a priori knowledge and schedule services accordingly. The system parameters available at particular location can be configured for that country or location. 
The channel sensing and estimation for the flight route will serve to establish channel statistics, as shown in Fig. 11. Aeronautical Channel Sensing. The CASDR cognitive channel awareness can configure the system to establish channel impairments for the flight route.

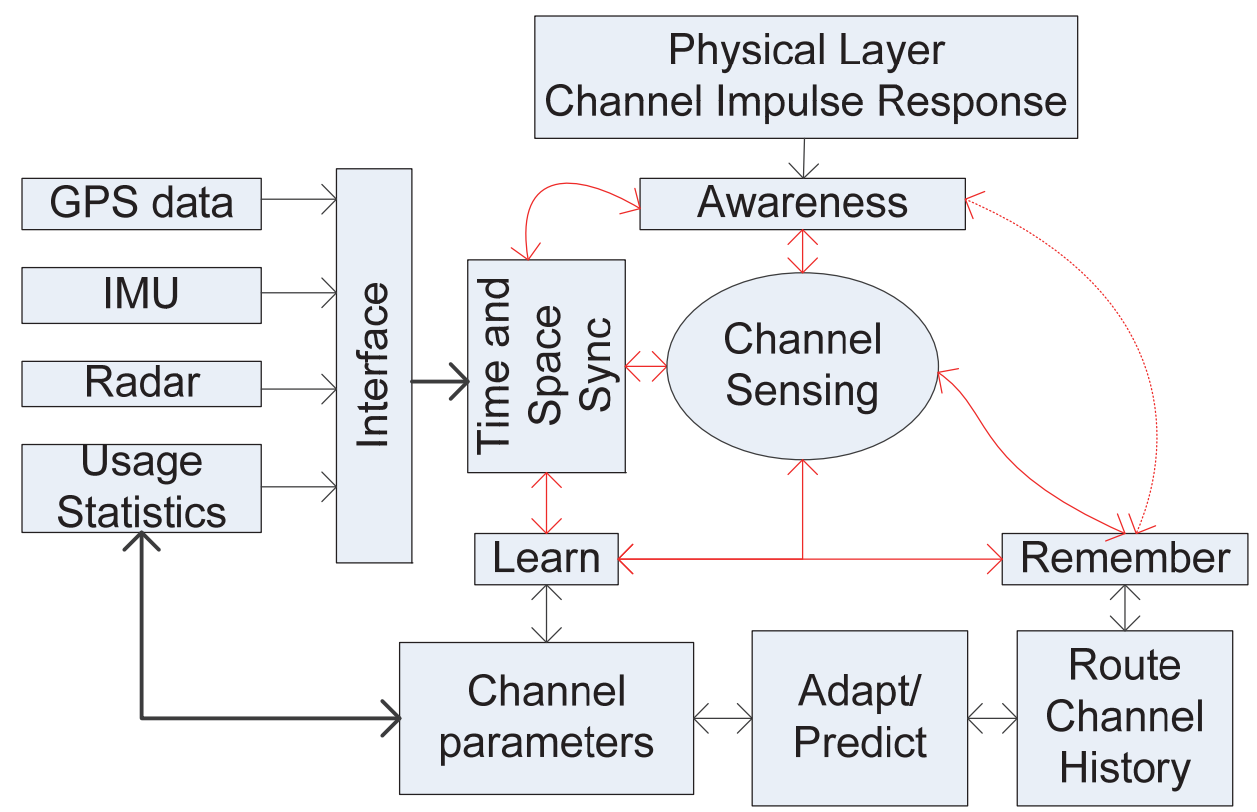

Fig. 11. Aeronautical Channel Sensing

- Remember:

System performance data gathered for different flight routes through different airlines will serve as means to remember these flight parameters, exchanged through a centralized data archives. Such data will grow in time and averaging over time will provide a reliable statistics for configuring the SDR radio parameters.

- Adapt and Predict:

The cognitive engine learning and sensing ability with an aircraft system will allow ability to predict system configuration parameters and adapt them to data gathered through flights travelled by other carriers.

\subsection{Aeronautical configurable hardware}

The key to a configurable system for an aeronautical system is to design hardware with minimum analog front-end, access different antenna system, digitize the signal and a scalable architecture. Fig. 12. Aeronautical Software Defined Radio presents such a system. The RF front-end board will support multiple bands with varying gain amplifiers. Closely coupled A/D boards with FPGAs are required for high-speed data connectivity and processing. A technique such as under sampling for demodulations is used to reduce the front-end components. The advances in ADC devices as well as non-compliance feature of Nyquist sampling theory is an enabler for an ASDR application. Violation of Nyquist theory will create signals aliased at integer multiple of sampling frequency $\left(\mathrm{N}^{*} \mathrm{fs}\right)$. This put the 
challenge on front-end processing system. The advances in programmable Digital Signal Processing (DSP) and Field Programmable Gate Array (FPGA) are ideal for such processing [Susaki]. FPGA offers ability to parallelize processing hence, allow a high-end processing throughput. The Virtex-6 FPGA family by Xilinx provides up to 2,016 DSP48 slices that deliver up to 1000 Giga MAC/s of DSP processing performance. Xilinx offers solutions for evolving standards such as WCDMA, WiMAX, TD_SCDMA and LTE. Texas instrument DSP products are now offering six DSP processors in a single package with processing capability 4000 million MACS (16-Bits) at $500 \mathrm{MHz}$.

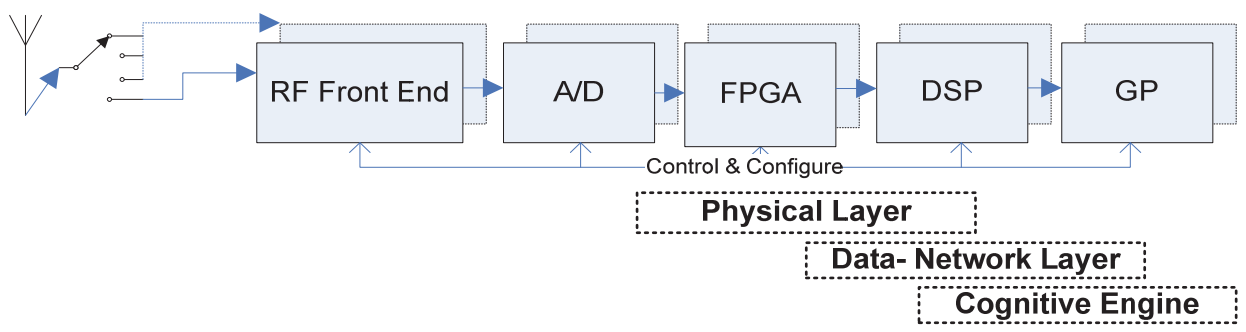

Fig. 12. Aeronautical Software Defined Radio

\section{Conclusions}

The advances in component technology, evolution in communication services, and increase in data demand and aircraft mobility creates an ideal application for CASDR to support the aeronautical system. Current deployed systems are beginning to form shape, i.e., gogo, however they are now adding another hardware box to provide connectivity. Since the system is hardwired for a particular modem, the evolution will require hardware modification to keep up with growth in the telecommunication growth. Accurate measurements of channel characteristics, such as Doppler, will allow spectral efficient modulation to be used for higher data rates. Advance algorithms along with processing capabilities can resolve the impact Doppler, due to aircraft high mobility. The novel cognitive channel measurement and estimation for each route will increase spectrum efficiency and in return provide high data throughput. An optimum combination of bandwidth, subcarrier bandwidth, acceptable Doppler frequency and multipath immunity system can be developed for ADN. This will result in an efficient use of the spectrum and provide a high data rate for the global connectivity.

\section{References}

AeroSat Corporation. (2008). About Airborne SatCom [Online]. Available: www.aerosat.com/about/ about_airborne_satcom.asp

Apostolis K. S., Hong N., \& P. Takis Mathiopoulos, ADC and DSP Challenges in the Development of Software Radio Base Stations.

Bello P. A., "Aeronautical channel characterization," IEEE Trans. communication., vol. COM21, pp. 548-563, May 1973. 
Blumenstein J. (2007). Aircell: Inflight Wi-Fi Built for the Airline Business [Online]. Available: blog.aircell.com.

Elnoubi, S.M., "A simplified stochastic model for the aeronautical mobile radio channel," Vehicular Technology Conference, 1992, IEEE 42nd , vol., no., pp.960-963 vol.2, 10-13 May 1992.

Eurocontrol, STATFOR - Air Traffic Statistics and Forecasts, http:/ / www.eurocontrol.int/statfor/

Haas, E., "Aeronautical channel modeling," Vehicular Technology, IEEE Transactions on, vol.51, no.2, pp.254-264, Mar 2002.

Hoeher, P., \& Haas, E., "Aeronautical channel modeling at VHF-band," Vehicular Technology Conference, 1999. VTC 1999 - Fall. IEEE VTS 50th, no., pp.1961-1966 vol.4, 1999.

Medina D., F. Hoffman, Ayaz, S., Rokitansky, C. H., Feasibilty of an Aeronautical Mobile Ad-Hoc Network Over the North Atlantic Corridor, Proceedings of IEEE Secon 2008.

Mohebbi B., E. C. Filho, R. Maestre, M. Davies, \& F. J. Kurdahi, “A case study of mapping a software-defined radio (SDR) application on a reconfigurable DSP core," in Proceedings of 1st IEEE/ACM/IFIP International Conference on Hardware/Software Codesign and System Synthesis, pp. 103-108,Newport Beach, Calif, USA, October 2003.

Mitola_1 J. and Gerald Q. Maguire, Jr, Cognitive Radio:Making Software Radios More Personal.

Mitola_2 J., The software Radio architecture, IEEE Commun. Mag, vol. 33, no. 5, pp. 26--38, May 1995.

NASA/CR-2008-215144, Future Aeronautical Communication Infrastructure Technology Investigation.

Newsky Project website: http://www.newsky-fp6.eu/.

Peter W., Glenister, S M., \& Alan W. Miller Motorola IEEE AES Systems Magazine, November 1999.

Salkintzis, A.K., Hong Nie, \& Mathiopoulos, P.T., “ADC and DSP challenges in the development of software radio base stations," IEEE Personal Communications, vol. 6, no. 4, pp. 47-55, Aug. 1999.

Sakhaee, E., \& Jamalipour, A., "The Global In-Flight Inter-net," Selected Areas in Communications, IEEE Journal on , vol.24, no.9, pp.1748-1757, Sept. 2006.

Srikanteswara R., Chembil Palat, R., Reed J. H., \& Athanas P., An Overview of Configurable Computing Machines for Software Radio Handsets, IEEE comms Mag., Vol.41 No.7, pp.134-141, July 2003.

Susaki H., "A Fast Algorithm for High-Accuracy Frequency Measurement: Application to Ultrasonic Doppler Sonar," in IEEE journal of oceanic engineering, vol. 27, no. 1, January 2002.

Zhang, Y., \& Nirwan Ansari, Wireless Telemedicine service over integrated IEEE802.11/Wlan and 802.16/Wimax, IEEE Wireless Communications, February 2010. 
Zanikopoulos A. Hegt, \& Van Roermund H., A. Programmable/Reconfigurable ADCs For Multi-standard Wireless Terminals, IEEE Conferences in Communications, Circuits and Systems Proceedings, 2006 International Conference, Volume: 2, 2006 , Page(s): 1337 $-1341$. 


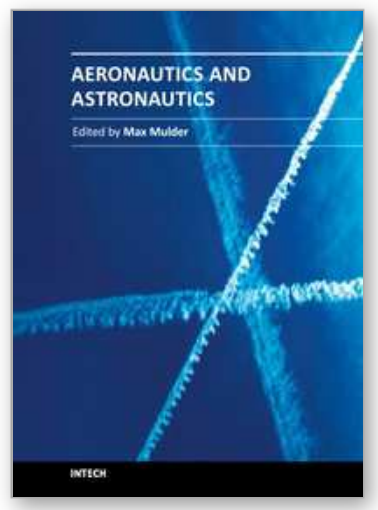

\author{
Aeronautics and Astronautics \\ Edited by Prof. Max Mulder
}

ISBN 978-953-307-473-3

Hard cover, 610 pages

Publisher InTech

Published online 12, September, 2011

Published in print edition September, 2011

In its first centennial, aerospace has matured from a pioneering activity to an indispensable enabler of our daily life activities. In the next twenty to thirty years, aerospace will face a tremendous challenge - the development of flying objects that do not depend on fossil fuels. The twenty-three chapters in this book capture some of the new technologies and methods that are currently being developed to enable sustainable air transport and space flight. It clearly illustrates the multi-disciplinary character of aerospace engineering, and the fact that the challenges of air transportation and space missions continue to call for the most innovative solutions and daring concepts.

\title{
How to reference
}

In order to correctly reference this scholarly work, feel free to copy and paste the following:

Mustafa Cenk Erturk, Wilfrido Moreno, Jamal Haque and Huseyin Arslan (2011). Aeronautical Data Networks, Aeronautics and Astronautics, Prof. Max Mulder (Ed.), ISBN: 978-953-307-473-3, InTech, Available from: http://www.intechopen.com/books/aeronautics-and-astronautics/aeronautical-data-networks

\section{INTECH}

open science | open minds

\section{InTech Europe}

University Campus STeP Ri

Slavka Krautzeka 83/A

51000 Rijeka, Croatia

Phone: +385 (51) 770447

Fax: +385 (51) 686166

www.intechopen.com

\section{InTech China}

Unit 405, Office Block, Hotel Equatorial Shanghai

No.65, Yan An Road (West), Shanghai, 200040, China

中国上海市延安西路65号上海国际贵都大饭店办公楼 405 单元

Phone: +86-21-62489820

Fax: +86-21-62489821 
(C) 2011 The Author(s). Licensee IntechOpen. This chapter is distributed under the terms of the Creative Commons Attribution-NonCommercialShareAlike-3.0 License, which permits use, distribution and reproduction for non-commercial purposes, provided the original is properly cited and derivative works building on this content are distributed under the same license. 